\title{
Inquiry of Water-Soluble Polysaccharide Extraction Conditions from Grapefruit Skin
}

\author{
Shiyu Tan, Qiliang Xu, Ziping Luo, Zilin Liu, Haiyan Yang, Lingli Yang \\ Institute of Chemistry and Chemical Engineering, Chongqing University, Chongqing, China \\ E-mail: tshy@cqu.edu.cn \\ Received August 23, 2011; revised September 30, 2011; accepted October 10, 2011
}

\begin{abstract}
Water-soluble polysaccharide was isolated from grapefruit with the method of water-dissolving and ethanolprecipitating. By single factor test and orthogonal experimental method, the extraction conditions of the water-soluble polysaccharide were investigated, which contained liquid-solid ratio, temperature, time and $\mathrm{pH}$. The results showed that $\mathrm{pH}$ was the most important factor of polysaccharide extraction, followed by temperature. The optimized extraction technology conditions were obtained. The liquid-solid ratio was 1:40, temperature was $90^{\circ} \mathrm{C}$, time was $2.0 \mathrm{~h}$, and $\mathrm{pH}$ was 2 . The highest extraction rate of crude polysaccharide was $8.30 \%$.
\end{abstract}

Keywords: Grapefruit, Water-Soluble Polysaccharide, Extracting Condition, Single Factor Experiment and Orthogonal Experiment

\section{Introduction}

Polysaccharide is an important bioactive substance with some physiological functions, such as immune, regulating cell growth and senescence [1]. Polysaccharide can be widely used in medicine, health products, materials and functional foods. As a green bio-medical product, it has a broad market prospect. Grapefruit is Rutaceae, and its skin accounts for about $40 \%$ of the total grapefruit. The bulk of the skins from agriculture or food processing not only cause tremendous waste of resources, but pollute the environment [2]. Grapefruit skin with potential value and application prospect is worth developing for us. Now the main deep processing of grapefruit skin are extractions of essential oil, pectin and pigment $[3,4]$, but its use value is far more than those.

Water-soluble polysaccharide is one of the important ingredients of grapefruit skin. The great majority exist with other polysaccharides or substances together by hydrogen bonding in the form of natural products. Li Xia, who studied the method of removing glycoprotein and determining polysaccharide content [5], had done watersoluble polysaccharide extraction experiment of grapefruit skin. But he did not consider the extraction technology conditions of polysaccharide.

In this paper, the process conditions of extraction of water-soluble polysaccharide from the grapefruit skin were studied and optimized. Water-soluble polysaccharide was gained by the method of hot water backflow extraction. Liquid-solid ratio, temperature, time and $\mathrm{pH}$ were respectively studied in the experiment. Crude polysaccharide was purified by Sevage method. The mass fraction of the polysaccharide was determined by phenolsulfuric acid method. And the extraction conditions were optimized by orthogonal test.

\section{Reagent and Equipments}

\subsection{Reagent}

All solvents used (absolute ethyl alcohol, concentrated sulfuric acid, phenol, acetone, n-butyl alcohol, chloroform) were of analytical reagent grade $(>99 \%)$.

\subsection{Equipments}

721 spectrophotometer vacuum oven (Shanghai TianPu analysis instrument Co., LTD), Electronic constant temperature stainless steel water bath pot (instrument Co., LTD of Shanghai Guangdi), Circulating water type multi-purpose vacuum pump (Industry \& trade Co., LTD of Zhengzhou Great Wall), LD4-2 low speed centrifuge (Beijing medical centrifuge plant), DZF-6021 type vacuum constant temperature drying oven (Shanghai Jing 
hong laboratory equipment Co., LTD), and so on.

\section{Experimental Methods}

\subsection{Extraction and Purification of Water-Soluble Polysaccharide from Grapefruit Skin}

Dried grapefruit skin was ground by blender machine. The grapefruit skin powder was weighed accurately and distilled water was solvent. We adopted different liquidsolid ratio, temperature, and time and $\mathrm{pH}$ in the polysaccharide extraction. After extraction, the extractive was filtrated with a Buchner funnel in vacuum and enriched to a definite volume subsequently. Then three times volume of anhydrous alcohol was added into the filtrate, and rough polysaccharide dissolved out in the form of floc precipitation, which was centrifuged at $4000 \mathrm{r} / \mathrm{min}$ for $15 \mathrm{~min}$ in a high-speed centrifuge. Rough polysaccharide contained protein impurities. They were removed by Sevage reagent [6], and pure polysaccharide was obtained after repeated removal. Since washed by anhydrous ethanol and acetone in turn, the sediment was finally dried in an oven at $80^{\circ} \mathrm{C}$ overnight.

\subsection{Determination of Mass Fraction of Polysaccharide}

\subsubsection{Preparation of Standard Curve}

Absorb glucose standard liquid (100 $\mu \mathrm{g} / \mathrm{ml})$ 0.1, 0.2, 0.4, $0.6,0.8,1.0,1.2 \mathrm{ml}$ into tubes. All was added distilled water to $2.0 \mathrm{ml}$. Then add phenol liquid (6\%) $1.0 \mathrm{ml}$ and concentrated sulfuric acid $5.0 \mathrm{ml}$. The mixed solution contained phenol liquid, sulfuric acid and a certain amount of glucose standard fluid was total $8.0 \mathrm{ml}$. Put them in a boiling water bath for heating $15 \mathrm{~min}$, and cool them to room temperature quickly. In blank, glucose standard liquid was substituted with $2.0 \mathrm{ml}$ distilled water. Then the glucose content of mixed solution was determined by phenol-sulfuric acid [7] method, at the maximum absorption wavelength $(490 \mathrm{~nm})$. With glucose mass concentration $(\mu \mathrm{g} / \mathrm{ml})$ for abscissa and absorbance value for $\mathrm{y}$-coordinate, standard curve was drawn.

\subsubsection{Determination of Conversion Factor}

$40 \mathrm{mg}$ refined polysaccharide was put into the measuring flask $(100 \mathrm{ml})$ and it was filled with distilled water. This was called reserve liquid. According to the method of standard curve preparation, absorbance value of reserve liquid $(0.2 \mathrm{ml})$ was determined. The content of glucose in polysaccharide reserve liquid $\left(\rho_{1}\right)$ was calculated by the standard curve. Conversion factor F was calculated according to the following Equation (1).

$$
F=m_{1} /\left(\rho_{1} \times D_{1}\right) .
$$

In the Equation (1): $m_{1}$ was polysaccharide quality $(\mu \mathrm{g})$ in reserve liquid $(0.2 \mathrm{ml}) . \rho_{1}$ was mass concentration $(\mu \mathrm{g} / \mathrm{ml})$ of glucose in polysaccharide liquid. $D_{1}$ was dilution factor of polysaccharide.

\subsubsection{Determination of Mass Fraction of Polysaccharide}

$10 \mathrm{mg}$ crude polysaccharide was weighed precisely and placed into the measuring flask with $100 \mathrm{ml}$ distilled water. The absorbance value of crude polysaccharide solution $(0.1 \mathrm{ml})$ was determined according to the method of standard curve preparation. Mass concentration $\left(\rho_{2}\right)$ of glucose was also evaluated according to the standard curve. Then polysaccharide content $W(\%)$ was calculated in Equation (2).

$$
W(\%)=\left(\rho_{2} \times D_{2} \times F \times 100\right) / m_{2} .
$$

In the Equation (2): $\rho_{2}$ was mass concentration $(\mu \mathrm{g} / \mathrm{ml})$ of glucose in crude polysaccharide liquid. $D_{2}$ was dilution factor of polysaccharide. $F$ was conversion factor. $m_{2}$ was the quality $(\mu \mathrm{g})$ of crude polysaccharide.

\subsection{Optimization of Extraction Condition of Water-Soluble Polysaccharide on the Basis of Orthogonal Test}

Select $\mathrm{L}_{9}\left(3^{4}\right)$ to do the orthogonal test. The selected technical parameters of the orthogonal test were liquidsolid ratio, temperature, time and $\mathrm{pH}$.

\section{Results and Discussion}

\subsection{Mass Fraction of Polysaccharide}

Absorbance value was determined at $490 \mathrm{~nm}$ by phenolsulfuric method. With glucose mass concentration for abscissa and absorbance value for $y$-coordinate, standard curve (as shown) was drawn. In Figure 1 the liner equation was $y=0.0573 x-0.0281, R^{2}=0.9966$.

Take the data $x_{1}=\rho_{1}=2.986 \mu \mathrm{g} / \mathrm{ml}$ into Equation (1), and the result was $F=0.6698$. And $x_{2}=\rho_{2}$ was $1.6 \mu \mathrm{g} / \mathrm{ml}$. Polysaccharide mass fraction $\mathrm{W}(\%)$ was calculated by Equation (2). $W(\%)$ was $85.73 \%$.

\subsection{Effect of Liquid-Solid Ratio}

The effect of liquid-solid ratio on extraction of watersoluble polysaccharide is illustrated in Figure 2. To determine the most appropriate sample to solvent ratio to extract polysaccharide, liquid-solid ratios of 1:10, 1:20, 1:30, 1:40 and 1:50 were tested, while other conditions, 


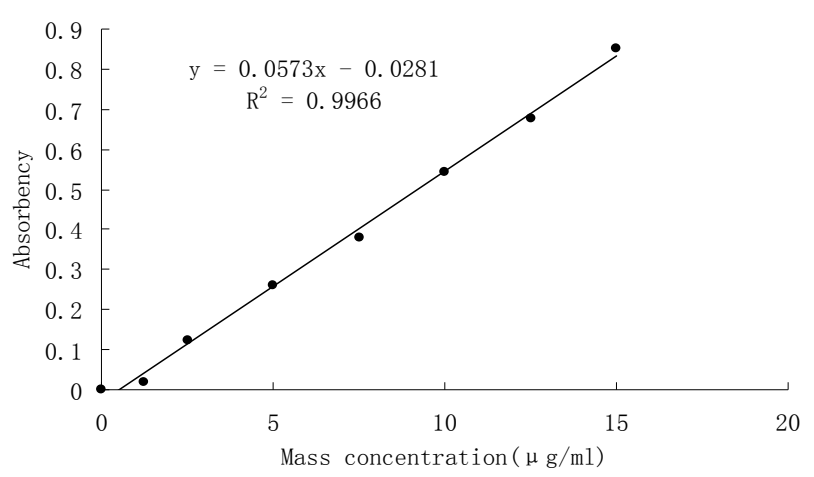

Figure 1. Standard curve of glucose.

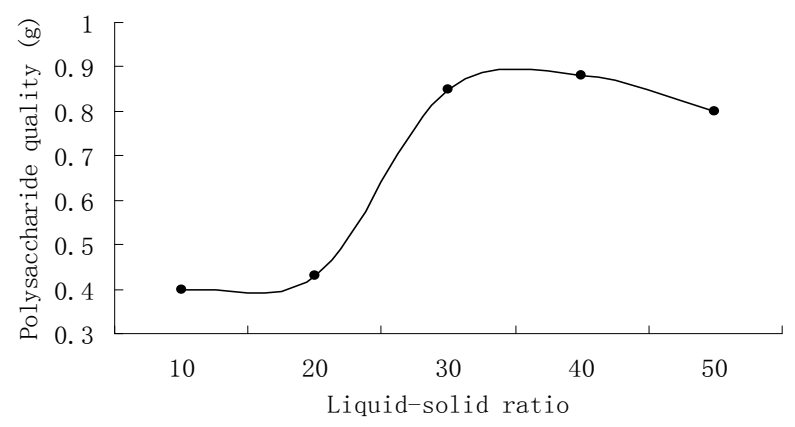

Figure 2. Effect of liquid-solid ratio on extraction of watersoluble polysaccharide.

such as the time for the experiment, the temperature, the $\mathrm{pH}$ and the experimental procedure, were kept the same. Figure 2 shows an increasing trend in the range of ratios from $1: 10$ to $1: 40$. But when in $1: 30$ and $1: 40$, the yield remained stable. The reason is when the dissolution of the polysaccharide reached a certain degree, the effect of liquid-solid ratio became weak. When liquid-solid was $1: 50$, the amount of polysaccharide began to reduce. This was due to dissolution of other impurities, which limited the dissolution of the polysaccharide. Higher liquid-solid ratio caused more energy consumption in the subsequent enrichment process, so the liquid-solid ratio was chosen appropriately around 1:40.

\subsection{Effect of Time}

To determine an appropriate time for maximum polysaccharide extraction, $0.5 \mathrm{~h}, 1.0 \mathrm{~h}, 1.5 \mathrm{~h}$, and $2.0 \mathrm{~h}$ and $2.5 \mathrm{~h}$ were chosen, while the other conditions were kept the same. Figure 3 indicates that the amount of polysaccharide increased with rise of extraction time from $0.5 \mathrm{~h}$ to $2.0 \mathrm{~h}$. That is because in a certain time range, the longer time is, the more polysaccharide dissolves. And the dissolution of polysaccharide appears to be equilibrium. But when the extraction period was further extended, the amount of polysaccharide decreased. The

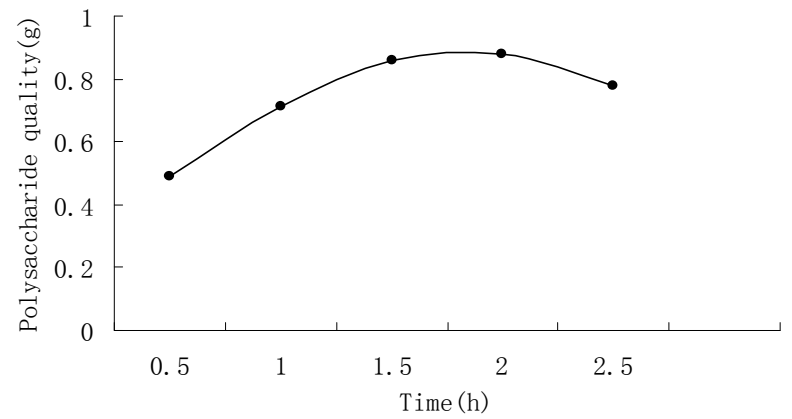

Figure 3. Effect of time on extraction of water-soluble polysaccharide.

decrease in the polysaccharide yield by increasing the extraction period to $2.5 \mathrm{~h}$ was due to the thermal degradation of the extracted polysaccharide. Considering energy and efficiency, extraction time was $2 \mathrm{~h}$.

\subsection{Effect of Temperature}

To get an appropriate temperature for maximum polysaccharide extraction, $55^{\circ} \mathrm{C}, 65^{\circ} \mathrm{C}, 75^{\circ} \mathrm{C}, 85^{\circ} \mathrm{C}$ and $95^{\circ} \mathrm{C}$ were chosen, while the other conditions were kept the same. In Figure 4, with the rise of temperature, the amount of polysaccharide gradually raised. This is related to the solubility of polysaccharide. It increases with the rise of temperature. But when temperature was nearly $100^{\circ} \mathrm{C}$, a lot of protein in the skin of grapefruit dissolved out. This increased workload to remove protein, and higher temperature also influenced the physiological activity of polysaccharide, even caused polysaccharide to degrade. So the appropriate extraction temperature was $95^{\circ} \mathrm{C}$.

\subsection{Effect of pH}

While the other conditions were kept the same, a $\mathrm{pH}$ range of 2 - 6 was chosen to determine an appropriate $\mathrm{pH}$ for maximum polysaccharide extraction. Figure 5 presents that the amount of polysaccharide extracted was found to increase with decreasing $\mathrm{pH}$. This is relevant to the structure of polysaccharide and the isoelectric point of protein. The high concentration of hydrogen ions in the solvent (at low $\mathrm{pH}$ ) stimulated the hydrolysis of an insoluble polysaccharide constituent. This will break the bonds between the polysaccharide molecule and the cell wall. When $\mathrm{pH}$ was higher, it promoted the dissolution of protein and inhibited the dissolution of polysaccharide. Therefore, in this study, greater amounts of polysaccharide had been extracted at $\mathrm{pH} 2$ compared with slightly higher $\mathrm{pHs}$. And the lower $\mathrm{pH}$ was good for extraction of polysaccharide in grapefruit skin. 


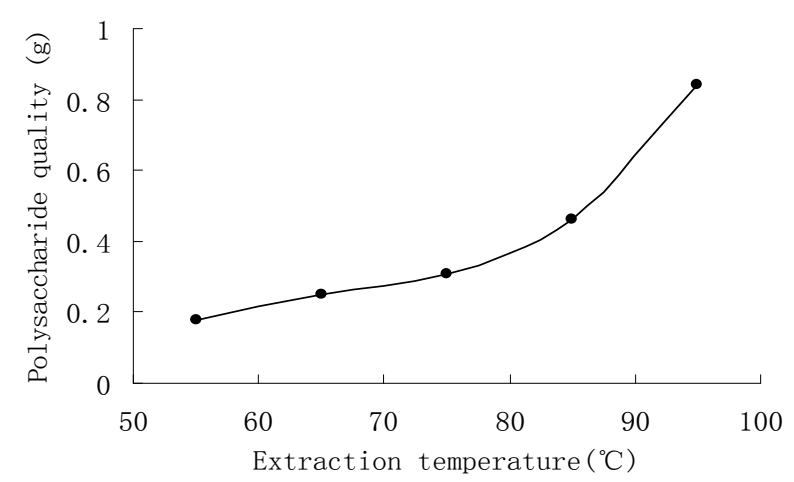

Figure 4. Effect of temperature on extraction of watersoluble polysaccharide.

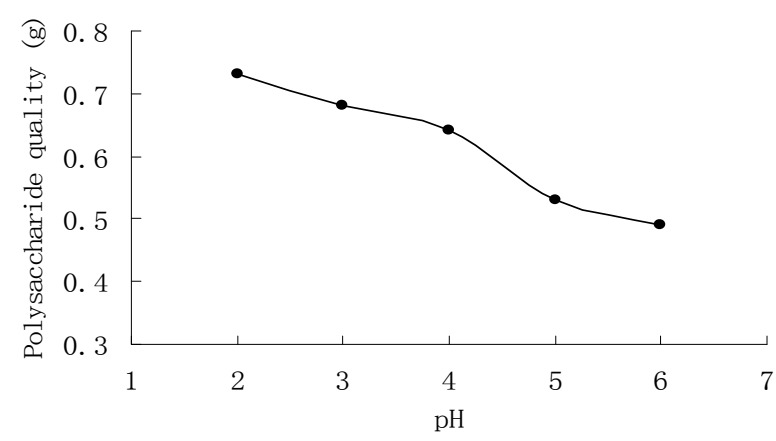

Figure 5. Effect of pH on extraction of water-soluble polysaccharide.

\subsection{Orthogonal Test Analysis}

On the basis of single factor experiment above, the ex- traction technology of water-soluble polysaccharide was optimized by orthogonal experiment. The extraction yields are different according to different liquid-solid ratio, time, $\mathrm{pH}$ and temperature. Therefore, factors were selected to determine the optimal extraction conditions based on the single factor experiment. The factors and experimental results were listed in Table 1. Liquid-solid ratios were 1:20, 1:30 and 1:40. pH was 2, 3 and 4. Extraction time was $0.5 \mathrm{~h}, 1.0 \mathrm{~h}$ and $2.0 \mathrm{~h}$. Temperature was $85^{\circ} \mathrm{C}, 90^{\circ} \mathrm{C}$ and $95^{\circ} \mathrm{C}$.

The results in Table 2 show that $\mathrm{pH}$ played the leading role in the polysaccharide extraction, followed by temperature and extraction time. We can summarize the optimized extraction conditions from the two tables. The liquid-solid ratio was $1: 40$. Temperature was $90^{\circ} \mathrm{C}$. Extraction time was $2.0 \mathrm{~h}$. pH was 2 . Under these conditions, the yield of polysaccharide of grapefruit skin was $8.30 \%$.

\section{Conclusions}

- $\mathrm{pH}$ was the most significant factor, followed by temperature and extraction time and the last was the liquid-solid ratio.

- The optimized extraction technology conditions: The liquid-solid ratio was $1: 40$, temperature was $90^{\circ} \mathrm{C}$, time was $2.0 \mathrm{~h}$, and $\mathrm{pH}$ was 2 .

- The highest extraction rate of water-soluble polysaccharide was $8.30 \%$.

- In the experiment, energy consumption and extraction efficiency should be considered in the choice of tem-

Table 1. Results and range analysis of orthogonal test on extraction conditions.

\begin{tabular}{cccccc}
\hline Test number & Liquid-solid & $\mathrm{pH}$ & Extraction time $(\mathrm{h})$ & Temperature & Polysaccharide yield \\
\hline 1 & $1: 20$ & 2 & 0.5 & 85 & $4.03 \%$ \\
2 & $1: 20$ & 3 & 1.0 & 90 & $3.41 \%$ \\
3 & $1: 20$ & 4 & 2.0 & 95 & $5.36 \%$ \\
4 & $1: 30$ & 2 & 1.0 & 90 & $3.73 \%$ \\
5 & $1: 30$ & 3 & 2.0 & 90 & $3.71 \%$ \\
6 & $1: 30$ & 4 & 0.5 & 95 & $8.30 \%$ \\
7 & $1: 40$ & 2 & 2.0 & 85 & $4.86 \%$ \\
9 & $1: 40$ & 3 & 0.5 & 0.5 & $3.55 \%$ \\
T1 & $1: 40$ & 4 & 1.0 & 0.363 & 0.513 \\
T2 & 0.423 & 0.600 & 0.417 & 0.527 & 0.164 \\
\hline
\end{tabular}


Table 2. Analysis of variance.

\begin{tabular}{ccccc}
\hline Factors & Bias squares & DOF & F & F (a=0.05) \\
\hline L-s ratio & 0.033 & 2 & 0.641 & 4.460 \\
$\mathrm{pH}$ & 0.080 & 2 & 1.553 & 4.460 \\
Time & 0.044 & 2 & 0.854 & 4.460 \\
Temperature & 0.049 & 2 & 0.951 & 4.460 \\
Error & 0.21 & 8 & & \\
\hline
\end{tabular}

perature and extraction time. Or it can change polysaccharide activity and cause the dissolution of impurities in the skin. This will adversely affect extraction and purification of polysaccharide.

- To the purified polysaccharide of grapefruit skin, its biological activity should be researched. The value of the water-soluble polysaccharide of grapefruit skin could be studied with some special properties [8,9] (such as film characteristic). For example, polysaccharide can be made into biodegradable film. This suggests that a process for the efficient utilization of the wastes in question is feasible.

\section{Acknowledgements}

This work was financially supported by the Chemistry and Chemical Engineering Institute of Chongqing University.

\section{References}

[1] H. L. Jin and Z. R. Xu, "Antiviral and Immune Research Progress of Polysaccharide," Feed Expo, Vol. 1, 2002, pp. 28-30.

[2] C. G. Qin, K. X. Huang and H. B. Xu, "Isolation and Characterization of Novel Polysaccharides from the $\mathrm{Mu}-$ cus of the Loach, Misgurnus Anguillicaudatus," Carbohydrate Polymer, Vol. 49, No. 3, 2002, pp. 367-371. doi:10.1016/S0144-8617(01)00335-6

[3] F. Yamaguchif, Y. Ota and C. Hatanaka, "Extraction and Purification of Pectic Polysaccharides from Soybean Okara and Enzymaticanalysis of Their Structures," Carbohydrate Polymer, Vol. 30, No. 4, 1996, pp. 265-273. doi:10.1016/S0144-8617(96)00046-X

[4] D. Y. Jia, K. Yao and M. Tan, "The Physiological Activity Component in Shaddock Are Reviewed," Food and Fermentation Industry, Vol. 27, No. 11, 2001, pp. 74-78.

[5] X. Li, "The Extraction and Test of Polysaccharide in Shaddock Ped," Value Engineering, Vol. 29, No. 18, 2010, pp. 255-256.

[6] Y. J. Fan, Q. Zhang and B. Zhu, "Study on Extraction of Soluble Soybean Polysaccharides," Food Science, Vol. 28, No. 9, 2007, pp. 295-298.

[7] Q. Dong, L. Y. Zheng and J. N. Fang, "Modified Phenol Sulfuric Acid Method for Determination of Content of Oligo and Polysaccharides," Chinese Pharmaceutical, Vol. 31, No. 9, 2001, pp. 550-553.

[8] X. H. Yue, "Research of Edible Chitosan and Starch Composite Membrane," Food Science, 2004, pp. 7-10.

[9] H. Q. Xu and Z. H. Wei, "Production Process of Water Insoluble Edible Film of Chitosan and Starch," Food Research and Development, Vol. 22, 2001, pp. 20-22. 